\title{
EIGENSHARP GRAPHS: DECOMPOSITION INTO COMPLETE BIPARTITE SUBGRAPHS
}

\author{
THOMAS KRATZKE ${ }^{1}$, BRUCE REZNICK ${ }^{2}$, AND DOUGLAS WEST ${ }^{3}$
}

\begin{abstract}
Let $\tau(G)$ be the minimum number of complete bipartite subgraphs needed to partition the edges of $G$, and let $r(G)$ be the larger of the number of positive and number of negative eigenvalues of $G$. It is known that $\tau(G) \geq r(G)$; graphs with $\tau(G)=r(G)$ are called eigensharp. Eigensharp graphs include graphs, trees, cycles $C_{n}$ with $n=4$ or $n \neq 4 k$, prisms $C_{n} \square K_{2}$ with $n \neq 3 k$, "twisted prisms" (also called "Möbius ladders") $M_{n}$ with $n=3$ or $n \neq 3 k$, and some Cartesian products of cycles.

Under some conditions, the weak (Kronecker) product of eigensharp graphs is eigensharp. For example, the class of eigensharp graphs with the same number of positive and negative eigenvalues is closed under weak products. If each graph in a finite weak product is eigensharp, has no zero eigenvalues, and has a decomposition into $\tau(G)$ stars, then the product is eigensharp. The hypotheses in this last result can be weakened. Finally, not all weak products of eigensharp graphs are eigensharp.
\end{abstract}

1. Introduction. Extremal graph theory abounds with the study of graph decomposition parameters. Given a family of graphs $\mathbf{F}$, let $\tau_{\mathbf{F}}(G)$ denote the minimum decomposition of $G$ into graphs from $\mathbf{F}$, i.e. the minimum number of edge-disjoint subgraphs of $G$ that belong to $\mathbf{F}$ and cover the edges of $G$. The parameter is welldefined as long as $K_{2} \in \mathbf{F}$, where $K_{n}$ denotes the complete graph or clique on $n$ vertices. The parameter $\tau_{\mathbf{F}}(G)$ has been studied for several families $\mathbf{F}$ :

\begin{tabular}{l|l}
$\mathbf{F}$ & $\tau_{\mathbf{F}}(G)$ \\
\hline complete graphs & $\begin{array}{l}\text { clique partition number } \\
\text { arboricity }\end{array}$ \\
forests & (see [6]) \\
paths and cycles & biparticity (see [4]) \\
all bipartite graphs & edge-chromatic number \\
matchings & vertex cover number \\
stars &
\end{tabular}

Note that more cliques may be needed to partition the edges of a graph than to cover them, as for the 4 -vertex graph with 5 edges, because subgraphs of cliques

Received by the editors January 26,1987 . Portions of this material were presented at the First International Conference on Graph Theory in Jinan, China, in June 1986.

1980 Mathematics Subject Classification (1985 Revision). Primary 05C35, 05C50, 05C75; Secondary $15 \mathrm{~A} 18$.

Key words and phrases. Decomposition, bipartite subgraph, graph product.

${ }^{1}$ Research supported by ONR Grant N00014-85K0570.

${ }^{2}$ Supported by the National Science Foundation and a fellowship from the Alfred P. Sloan Foundation.

${ }^{3}$ Research supported in part by ONR Grant N00014-85K0570 and by NSF grant DMS 8504322 . 
need not be cliques. Arboricity is mostly of interest because there is a min-max formula for it, and it generalizes to the setting of matroids. Lovász's motivation in [6] was the long-standing conjecture of Gallai that every $n$-vertex graph can be decomposed into $\lceil n / 2\rceil$ paths; Lovász proved that $\lfloor n / 2\rfloor$ paths and/or cycles will suffice. Harary, Hsu, and Miller [4] proved that for every graph $G$ the minimum number of bipartite graphs that partition its edges is exactly $\left\lceil\log _{2} \chi(G)\right\rceil$, where $\chi(G)$ denotes the ordinary chromatic number. The edge-chromatic number and vertex cover number (minimum number of vertices needed to have an endpoint of each edge) are well-studied parameters with many applications. Note that for most of these decomposition parameters, $\mathbf{F}$ is a class of bipartite graphs.

In this paper, we let $\mathbf{F}$ be the collection of all complete bipartite graphs $K_{r, s}$ and write $\tau(G)=\tau_{\mathbf{F}}(G)$. One motivation for studying this parameter is minimizing storage space; listing the subgraphs in a minimum complete bipartite decomposition of $G$ never takes more space than the adjacency list representation. When Graham and Pollak [3] first studied this parameter for the complete graph, they were motivated by a network addressing problem.

The complete bipartite graphs $K_{1, r}$ are called stars; we say that a star is "centered" on its single vertex of high degree. Because every star is a complete bipartite graph, the vertex cover number of $G$ is an upper bound on $\tau(G)$; given any vertex set $U$ forming a vertex cover of $G$, we can partition the edges of $G$ using stars centered at the vertices of $U$. Note that a set of vertices forms a vertex cover if and only if the remaining vertices form an independent (pairwise nonadjacent) set of vertices. The maximum size of an independent set of vertices is called the independence number, is written $\alpha(G)$, and is more widely studied than the vertex cover number. Restricting $\mathbf{F}$ to stars gives the bound $\tau(G) \leq n-\alpha(G)$ for a graph $G$ on $n$ vertices; Erdős conjectured that $\tau(G)=n-\alpha(G)$ for almost all graphs.

In general, the independence number is a difficult (NP-complete) graph parameter to compute. In this paper, we will study classes of graphs for which $\tau(G)$ is easy to compute. Like $\alpha(G), \tau(G)$ is hard to compute in general. In fact, let $\mathbf{G}$ be the class of graphs without 4-cycles; we have $\tau(G)=n-\alpha(G)$ for all $G \in \mathbf{G}$, since graphs in $\mathbf{G}$ have no complete bipartite subgraphs other than stars. Therefore, computing $\tau(G)$ is equivalent to computing $\alpha(G)$ for graphs in G. Since testing $\alpha(G) \leq k$ (with inputs $G$ and $k$ ) is NP-complete even under the restriction $G \in \mathbf{G}$, testing $\tau(G) \leq k$ is also NP-complete given $G \in \mathbf{G}$. (We thank Lex Schrijver for pointing out that $\alpha(G) \leq k$ is NP-complete when restricted to $G \in \mathbf{G}$. Consider replacing each edge in arbitrary input $G$ by a path of three edges. The new graph $G^{\prime}$ has no 4-cycles, and $\alpha(G) \leq k$ if and only if $\alpha\left(G^{\prime}\right) \leq k+\|G\|$, where $\|G\|$ denotes the number of edges in $G$. Hence any polynomial algorithm to compute $\alpha$ for $G \in \mathbf{G}$ yields a polynomial algorithm to compute $\alpha$ for arbitrary graphs, but the latter is well known to be NP-complete.)

The first graphs for which $\tau(G)$ was studied were the complete graphs, which have plenty of 4-cycles. Nevertheless, for $G=K_{n}$ it still holds that $\tau(G)=n-\alpha(G)=$ $n-1$. Graham and Pollak [3] proved this algebraically. Tverberg [10] found a shorter proof using linear and quadratic equations, obtained independently by Lovász [7]. G. W. Peck [8] gave a similar proof using matrix ranks; no purely combinatorial short proof is known. 
Each of these proofs generalizes to arbitrary graphs, yielding a uniform lower bound on $\tau(G)$ in terms of the eigenvalues of $G$ (i.e., the eigenvalues of its adjacency matrix $A(G)$ ). Let $p(G), s(G), q(G)$ denote the number of positive, zero, and negative eigenvalues of $G$, respectively; $(p(G), s(G), q(G))$ is called the signature of $G$. The full list of $n$ eigenvalues of $A(G)$ is called the spectrum of $G$, written $\operatorname{Spec}(G)$. Letting $r(G)=\max \{p(G), q(G)\}$, the eigenvalue bound is $\tau(G) \geq r(G)$. For completeness, in $\S 2$ we provide the straightforward generalization of Tverberg's proof [10] to arbitrary graphs. At about the same time as [3], Hoffman [5] proved $\tau(G) \geq q(G)$ for arbitrary graphs, by yet another method.

When the eigenvalue bound is sharp, i.e. $\tau(G)=r(G)$, we say that $G$ is eigensharp. As suggested earlier, we can compute $\tau(G)$ for eigensharp graphs, because we can compute $r(G)$ for arbitrary graphs, assuming sufficient precision in arithmetic. Our results include proving that various classes of graphs are eigensharp and proving a sufficient condition for a type of product graph to be eigensharp. We pause to introduce concepts of graph products and related notation.

We use $V(G)$ and $E(G)$ to denote the vertex set and edge set of a graph $G$. We write $u \leftrightarrow v$ when vertices $u$ and $v$ are adjacent, and we let $\operatorname{Adj}(v)$ denote the set of neighbors of $v$. When we want to indicate the complete bipartite graph with specified partite sets $S, T$ of unspecified size, we write $K_{S, T}$.

There are three related concepts of graph product in common use: the weak product, the cartesian product, and the strong product. Selection of notation for these products is a long-standing controversy. A scheme due to Nesetril is logical. He denotes the products by $G \times H, G \square H$, and $G \otimes H$, respectively, by the mnemonic that these symbols are pictures of the three products of $K_{2}$ with itself. We adopt the $\square$ for cartesian product. However, the symbol $\times$ is so firmly associated with cartesian product in many minds that we use $*$ for weak product to emphasize the distinction, reserving $\times$ for the cartesian product of sets.

The weak product $G * H$ of $G$ and $H$ has vertex set $V(G * H)=V(G) \times V(H)$, with $(x, y) \leftrightarrow\left(x^{\prime}, y^{\prime}\right)$ in $G * H$ if $x \leftrightarrow x^{\prime}$ in $G$ and $y \leftrightarrow y^{\prime}$ in $H$. The weak product is also called the Kronecker product due to its effect on the adjacency matrices. When $A, B$ are $m$ by $n$ and $p$ by $q$ matrices, the Kronecker product $A \otimes B$ is an $m p$ by $n q$ matrix consisting of blocks $a_{i j} B$. When $A$ and $B$ are adjacency matrices, every block is $B$ or $\mathbf{0}$. In other words, $A(G * H)=A(G) \otimes A(H)$. The weak product is associative, so $G_{1} * \cdots * G_{k}$ is well defined.

The cartesian product $G \square H$ also has vertex set $V(G) \times V(H)$, but now $(x, y) \leftrightarrow$ $\left(x^{\prime}, y^{\prime}\right)$ in $G \square H$ if $x=x^{\prime}$ and $y \leftrightarrow y^{\prime}$, or if $x \leftrightarrow x^{\prime}$ and $y=y^{\prime}$. The strong product $G \otimes H$, which we will not use, has $(x, y) \leftrightarrow\left(x^{\prime}, y^{\prime}\right)$ if $x^{\prime}$ is adjacent or equal to $x$ and $y^{\prime}$ is adjacent or equal to $y$.

In $\S 3$ we show that all trees are eigensharp, the $n$-cycle $C_{n}$ is eigensharp precisely when $n=4$ or $n \neq 4 k$, the prism $C_{n} \square K_{2}$ is eigensharp if and only if $n \neq 3 k$, and various cycle products $C_{m} \square C_{n}$ are eigensharp. Also, the "Möbius ladder" $M_{n}$ is obtained from the prism $C_{n} \square K_{2}$ by replacing the two copies of one cycle edge by the other possible pair of independent edges; $M_{n}$ is eigensharp if and only if $n=3$ or $n \neq 3 k$.

In $\S 4$ we obtain several sufficient conditions for the weak product of graphs to be eigensharp; the main result supersedes [9]. It is easy to show that the class of eigensharp graphs with the same number of positive and negative eigenvalues is 
closed under weak products. The result of [9] amounts to the statement that weak products of complete graphs are eigensharp; we extend this to a class containing the complete graphs. If $G$ can be covered (optimally) by $\tau(G)$ stars, we say that $G$ is star-coverable. Let $\mathbf{H}$ be the collection of eigensharp star-coverable graphs with no zero eigenvalues. By a straightforward generalization of the argument in [9], we prove that any finite weak product of graphs in $\mathbf{H}$ is eigensharp, although it need not be in $\mathbf{H}$. We further strengthen this theorem to a larger class than $\mathbf{H}$, with a weaker defining condition. All the proofs are constructive, with explicit recipes given for forming optimal decompositions of the product from optimal decompositions of the factors. The investigation of this weaker criterion leads to an example of a pair of eigensharp graphs whose weak product is not eigensharp. We note here a correction to [9], which contains the remark that $C_{5} * C_{5}$ is not eigensharp. In fact, $C_{5}$ belongs to $\mathbf{H}$, and hence $C_{5} * C_{5}$ is eigensharp.

In order to compute $r(G * H)$, we need to know how eigenvalues are affected by taking the Kronecker product. If $\operatorname{Spec}(G)=\left(\lambda_{1}, \ldots, \lambda_{m}\right)$ and $\operatorname{Spec}(H)=$ $\left(\mu_{1}, \ldots, \mu_{n}\right)$, then $\operatorname{Spec}(G * H)$ is the collection $\left\{\lambda_{r} \mu_{s}: 1 \leq r \leq m, 1 \leq s \leq n\right\}$. This can be proved by exhibiting a full set of eigenvectors corresponding to these eigenvalues. If $\left\{\bar{u}^{r}\right\}$ and $\left\{\bar{v}^{s}\right\}$ are full sets of eigenvectors for $A(G)$ and $A(H)$ corresponding to the spectra above, then the vector $\bar{w}^{r s}$ whose entry in position $i, j$ is $u_{i}^{r} v_{j}^{s}$ satisfies $(A \otimes B) \bar{w}^{r s}=\left(\lambda_{r} \mu_{s}\right) \bar{w}^{r s}$, and the $\bar{w}$ 's can be shown to be independent (see $[1, \S 2.5]$ ).

The cartesian product affects eigenvalues in a similar way. If $\operatorname{Spec}(G)=\left(\lambda_{1}, \ldots\right.$, $\left.\lambda_{m}\right)$ and $\operatorname{Spec}(H)=\left(\mu_{1}, \ldots, \mu_{n}\right)$, then $\operatorname{Spec}(G \square H)$ consists of all $m n$ sums $\left\{\lambda_{r}+\right.$ $\left.\mu_{s}: 1 \leq r \leq m, 1 \leq s \leq n\right\}$. This is proved using the same eigenvectors $\bar{w}^{r s}$ as above (see $[\mathbf{1}, \S 2.5])$.

2. The eigenvalue lower bound. For completeness, we give the generalization of Tverberg's proof.

THEOREM $1[\mathbf{3}, \mathbf{7}, \mathbf{8}, \mathbf{1 0}] . \tau(G) \geq \max \{p, q\}$, where $(p, s, q)$ is the signature of $G$.

ProOF. When $G$ is decomposed into subgraphs $G_{1}, \ldots, G_{t}$, we may write $A(G)=\sum_{i=1}^{t} B_{i}$, where $B_{i}$ is the adjacency matrix of the subgraph obtained from $G_{i}$ by including any omitted vertices of $G$ as isolated vertices. Consider the associated quadratic forms, with variables $\bar{x}^{T}=\left(x_{1}, \ldots, x_{n}\right)$. If $G_{i}=K_{S, T}$, we have $\bar{x}^{T} B_{i} \bar{x}=2 \sum_{j \in S} x_{j} \sum_{k \in T} x_{k}$. Writing these linear expressions as $u=\sum_{j \in S} x_{j}$ and $v=\sum_{k \in T} x_{k}$, we have $\bar{x}^{T} B_{i} \bar{x}=2 u v=L^{2}(\bar{x})-M^{2}(\bar{x})$, where $L=z^{-1 / 2}(u+v)$ and $M=z^{-1 / 2}(u-v)$ are linear combinations of the variables $x_{1}, \ldots, x_{n}$. If $G$ is decomposable into $t$ complete bipartite subgraphs, applying this to each yields $\bar{x}^{T} A(G) \bar{x}=\sum_{i=1}^{t} L_{i}^{2}(\bar{x})-\sum_{i=1}^{t} M_{i}^{2}(\bar{x})$.

On the other hand, the adjacency matrix is a real symmetric matrix and thus has a full set of orthonormal eigenvectors $\left\{\bar{w}_{i}\right\}$. In other words, we can write $\bar{x}^{T} A(G) \bar{x}=\bar{x}^{T} Q \Lambda Q^{T} \bar{x}$, where $\Lambda$ is the diagonal matrix of eigenvalues $\lambda_{1} \geq \cdots \geq$ $\lambda_{n}$ and $Q$ has columns $\bar{w}_{1}, \ldots, \bar{w}_{n}$. Since $A(G)$ has $p$ positive and $q$ negative eigenvalues, we can write this as $\bar{x}^{T} A(G) \bar{x}=\sum_{i=1}^{p}\left(\bar{y}_{i} \cdot \bar{x}\right)^{2}-\sum_{i=n-q+1}^{n}\left(\bar{z}_{i} \cdot \bar{x}\right)^{2}$, where each $y_{i}$ or $z_{i}$ is $\sqrt{\left|\lambda_{i}\right|} \bar{w}_{i}$. 
Setting each $L_{i}(\bar{x})=0$ and $\bar{z}_{i} \cdot \bar{x}=0$ yields $t+q$ linear equations in $n$ variables. Also, note that $\bar{w}_{p+1}, \ldots, \bar{w}_{n-q}$ are the $s$-eigenvectors corresponding to the eigenvalue 0 , if $p+q<n$. We require also that $\bar{w}_{i} \cdot \bar{x}=0$ for $p<i \leq n-q$. If $t<p$, then $t+q+s<p+q+s=n$, and these equations have a nonzero simultaneous solution $\bar{x}=\bar{x}_{0}$. Setting $\bar{x}=\bar{x}_{0}$ in the two expressions for $\bar{x}^{T} A(G) \bar{x}$ yields $\sum_{i=1}^{p} y_{i}^{2}\left(\bar{x}_{0}\right)=-\sum_{i=1}^{t} M_{i}^{2}\left(\bar{x}_{0}\right)$. Since $\bar{x}_{0}$ is orthogonal to all eigenvectors with nonpositive eigenvalues, this equates a positive and a nonpositive number. We conclude $t \geq p ;$ similarly, $t \geq q$.

3. Examples of eigensharp graphs. It is well known that $\operatorname{Spec}\left(K_{n}\right)=$ $(n-1,-1, \ldots,-1)\left(\right.$ since $A\left(K_{n}\right)=J_{n}-I_{n}$ and $\operatorname{Spec}\left(J_{n}\right)=(n, 0, \ldots, 0)$ ), and hence the decomposition of $K_{n}$ into $n-1$ stars shows it is eigensharp. In discussing the eigensharpness of other graphs, we use two elementary remarks.

REMARK 1. If $\tau(G)=\left\lceil\frac{1}{2}(n-s(G))\right\rceil$, then $G$ is eigensharp.

REMARK 2. The $i$ th component of $A(G) \bar{x}$ is $\sum_{j \leftrightarrow i} x_{j}$; hence $\lambda$ is an eigenvalue of $G$ if and only if there are vertex weights such that $\sum_{j \leftrightarrow i} x_{j}=\lambda x_{i}$ for all $i$. In particular, $s(G)$ is the dimension of the space of solutions to $\sum_{j \leftrightarrow i} x_{j}=0$ for all $i$.

In the remainder of this section, we consider cycles, trees, prisms, and several other classes of graphs.

THEOREM 2. The cycle $C_{n}$ is eigensharp unless $n=4 k$ with $k \geq 2$.

PROOF. Denote the vertices of $C_{n}$ by the integers $\bmod n$, and let $\omega$ be a primitive $n$th root of unity. Define weights $\bar{z}_{j}$ by giving weight $\omega^{i j}$ to vertex $i$. Then $A \bar{z}_{j}=$ $\left(\omega^{j}+\omega^{-j}\right) \bar{z}_{j}$. Hence $\operatorname{Spec}\left(C_{n}\right)=\{2 \cos (2 \pi j / n): 0 \leq j<n\}$, as is well known [1]. In particular, $s\left(C_{n}\right)=2$ if $n=4 k$, otherwise $s\left(C_{n}\right)=0$. Furthermore, the only complete bipartite subgraphs are paths of one or two edges unless $n=4$, yielding $\tau(G)=\lceil n / 2\rceil$ unless $n=4$. By Remark $1, C_{n}$ is eigensharp unless $n=4 k$ for $k \geq 2$.

Next we consider trees and the implications of Remark 2. It is only possible to have $\tau(G)=\frac{1}{2}(n-s(G))$ when $p(G)=q(G)$. For bipartite graphs, such as trees, we do have $p(G)=q(G)$. By the example of $C_{8}$, not all bipartite graphs are eigensharp. However, we show next that $\tau(G)=\frac{1}{2}(n-s(G))$ when $G$ is a tree, and hence all trees are eigensharp. There are two workable approaches. Cvetković and Gutman [2] showed that the maximum size of a matching in a tree is $n-s(T)$. Constructing a decomposition into stars centered at one vertex from each edge of a maximum matching is one way to show $\tau(G)=\frac{1}{2}(n-s(T))$. Instead, we prove this directly by induction, which can also be viewed as a proof of the Cvetković-Gutman result.

\section{THEOREM 3. Every tree is eigensharp.}

PROOF. It suffices to prove $\tau(T)=\frac{1}{2}(n-s(T))$; we use induction on $n$. For the basis consider the star $K_{1, r}$; its spectrum is $(\sqrt{r}, 0, \ldots, 0,-\sqrt{r})$, and thus $\tau\left(K_{1, r}\right)=$ $1=\frac{1}{2}(n-s(T))$. If $T$ is not a star, consider a leaf at the end of a maximum-length path; call it vertex 1 . Let its neighbor be $v$, let $1, \ldots, k$ be the leaves adjacent to $v$, and let $w$ be the unique nonleaf adjacent to $v$. Let $\bar{z}$ be a solution to $A(T) \bar{x}=\overline{0}$. Examining vertices 1 and $v$ shows that $z_{v}=0$ and $\sum_{i=1}^{k} z_{i}=-z_{w}$. Because $z_{v}=0$, deleting $\{v, 1, \ldots, k\}$ leaves a smaller tree $T^{\prime}$ along with a corresponding vector $\bar{z}^{\prime}$ 
in the nullspace of $A\left(T^{\prime}\right)$. On the other hand, any vector $\bar{z}^{\prime}$ in this nullspace can be augmented to a vector solving $A(T) \bar{x}=\overline{0}$ by adding variables $z_{v}$ and $z_{1}, \ldots, z_{k}$ satisfying $z_{v}=0$ and $\sum_{i=1}^{k} z_{i}=-z_{w}$. Hence $s(T)=s\left(T^{\prime}\right)+k-1$. By the induction hypothesis, $\tau\left(T^{\prime}\right)=\frac{1}{2}\left(n-k-1-s\left(T^{\prime}\right)\right)$. Since any decomposition of $T^{\prime}$ can be augmented to a decomposition of $T$ by adding a star at $v, \tau(T) \leq 1+\tau\left(T^{\prime}\right)=$ $\frac{1}{2}(n-s(T))$. Because $T$ is bipartite, $r(T)=\frac{1}{2}(n-s(T))$, so equality holds.

THEOREM 4. The prism $C_{n} \square K_{2}$ is eigensharp unless $n=3 k$.

ProOF. As noted earlier, $\operatorname{Spec}\left(C_{n}\right)=\{2 \cos (2 \pi j / n): 0 \leq j<n\}$ and $\operatorname{Spec}\left(K_{2}\right)$ $=\{1,-1\}$, so $\operatorname{Spec}\left(C_{n} \square K_{2}\right)=\{2 \cos (2 \pi j / n) \pm 1\}$. For $0 \leq j<n$, the contribution of $j$ to the signature of $C_{n} \square K_{2}$ is $(0,0,2),(0,1,1),(1,0,1),(1,1,0)$, or $(2,0,0)$ when $\cos (2 \pi j / n)$ belongs to the set $\left[-1,-\frac{1}{2}\right),\left\{-\frac{1}{2}\right\},\left(-\frac{1}{2}, \frac{1}{2}\right),\left\{\frac{1}{2}\right\}$, or $\left(\frac{1}{2}, 1\right]$, respectively. How many $\cos (2 \pi j / n)$ 's fall in each set depends on the congruence class of $n \bmod 6$. The table below summarizes the cases.

\begin{tabular}{l|cccccc}
\multicolumn{7}{c}{ Spectrum of $C_{n} \square K_{2}$} \\
$n=$ & $6 k$ & $6 k+1$ & $6 k+2$ & $6 k+3$ & $6 k+4$ & $6 k+5$ \\
\hline$p$ & $n-2$ & $n+1$ & $n$ & $n-1$ & $n$ & $n+1$ \\
$s$ & 4 & 0 & 0 & 2 & 0 & 0 \\
$q$ & $n-2$ & $n-1$ & $n$ & $n-1$ & $n$ & $n-1$
\end{tabular}

Now consider the decomposition of $C_{n} \square K_{2}$ into complete bipartite subgraphs. We can decompose $C_{n} \square K_{2}$ into $n$ stars if $n$ is even, $n+1$ stars if $n$ is odd, as follows. Suppose the vertices of $C_{n}$ are $\{0, \ldots, n-1\}$ in cyclic order and those of $K_{2}$ are $\{u, v\}$, and describe the vertices of $C_{n} \square K_{2}$ as $\left\{u_{0}, \ldots, u_{n-1}\right\} \cup\left\{v_{0}, \ldots, v_{n-1}\right\}$. For the decomposition, use stars centered at $\left\{u_{i}: i\right.$ is even $\} \cup\left\{v_{i}: i\right.$ is odd $\}$. If $n$ is odd, this leaves the edge $\left(v_{1}, v_{n-1}\right)$ uncovered, so we include that as an additional subgraph. This decomposition shows $\tau\left(C_{n} \square K_{2}\right) \leq 2\lceil n / 2\rceil$, which equals $r\left(C_{n} \square K_{2}\right)$ in the table above unless 3 divides $n$, so $C_{n} \square K_{2}$ is eigensharp when 3 does not divide $n$.

We now show that $\tau\left(C_{n} \square K_{2}\right) \geq n$ for all $n$, proving that $C_{3 k} \square K_{2}$ is not eigensharp. Since $C_{n} \square K_{2}$ has $3 n$ edges, it suffices to show that the subgraphs in any decomposition contribute at most an average of 3 edges each. The only complete bipartite subgraphs of $C_{n} \square K_{2}$ having more than 3 edges are 4-cycles. Suppose the decomposition uses the 4-cycle $\left(u_{i-1}, v_{i-1}, v_{i}, u_{i}\right)$. Then the edges $\left(v_{i}, v_{i+1}\right)$ and $\left(u_{i}, u_{i+1}\right)$ must belong to stars in the decomposition. These stars together contribute at most 5 edges; i.e. a 4-cycle and the two stars forced on the higherindexed edges neighboring it cover at most 9 edges together. To counterbalance the 4-cycles we always take stars in the direction of increasing subscript, so we have partitioned the subgraphs of an arbitrary decomposition into sets for which the number of edges contributed is at most 3 times the number of subgraphs.

The Möbius ladder $M_{n}$ (so named in [1, p. 75]) is isomorphic to $C_{n} \square K_{2}$ except that in place of the edges $\left(u_{n}, u_{0}\right)$ and $\left(v_{n}, v_{0}\right)$ we have $\left(u_{n}, v_{0}\right)$ and $\left(v_{n}, v_{0}\right)$. In other words, $C_{n} \square K_{2}$ looks like a cylindrical band, and $M_{n}$ looks like a band with a twist. Not surprisingly, the results are similar to the results for $C_{n} \square K_{2}$. The spectrum of $M_{n}$, like those mentioned earlier, is computed in [1], but again we include a computation for completeness.

THEOREM 5. The Möbius ladder $M_{n}$ is eigensharp unless $n=3 k$ with $k \geq 2$. 
PrOOF. To compute $\operatorname{Spec}\left(M_{n}\right)$, view the edges along the rim of the ladder as a cycle of length $2 n$. The "rungs" formed by the edges $\left(u_{i}, v_{i}\right)$ become chords joining opposite vertices (separated by $n$ ) on the cycle. Let $\omega$ be a primitive $2 n$th root of unity, and let $\bar{z}_{j}=\left(1, \omega^{j}, \ldots, \omega^{(2 n-1) j}\right)$ be the $j$ th eigenvector of $C_{2 n}$ as discussed earlier. Then $A\left(M_{n}\right) \bar{z}_{j}=\left(\omega^{j}+\omega^{-j}+\omega^{n j}\right) \bar{z}_{j}$. Hence $\left\{\bar{z}_{j}\right\}$ are also the eigenvectors of $M_{n}$ (or, in fact, any circulant graph), and $\operatorname{Spec}\left(M_{n}\right)=\left\{2 \cos (2 \pi j / 2 n)+(-1)^{j}\right.$ : $0 \leq j \leq 2 n-1\}$. As in the preceding proof, we analyze by the congruence class of $n \bmod 6$ to get the following table.

\begin{tabular}{l|cccccc}
\multicolumn{7}{c}{ Spectrum of $M_{n}$} \\
$n=$ & $6 k$ & $6 k+1$ & $6 k+2$ & $6 k+3$ & $6 k+4$ & $6 k+5$ \\
\hline$p$ & $n-1$ & $n$ & $n-1$ & $n-2$ & $n+1$ & $n$ \\
$s$ & 2 & 0 & 0 & 4 & 0 & 0 \\
$q$ & $n-1$ & $n$ & $n+1$ & $n-2$ & $n-1$ & $n$
\end{tabular}

For the decomposition, again use stars at $\left\{u_{i}: i\right.$ is even $\} \cup\left\{v_{i}: i\right.$ is odd $\}$. If $n$ is even, this leaves the edge $\left(u_{n-1}, v_{0}\right)$ uncovered, so we include that as an additional subgraph. Hence we have $\tau\left(M_{n}\right) \leq 1+2\lfloor n / 2\rfloor$, which equals $r\left(M_{n}\right)$ as found in the table unless 3 divides $n$. The argument allowing at most 3 edges per subgraph in $C_{n} \square K_{2}$ is local and thus still applies here when $n \geq 4$ by exchanging $u_{i}$ and $v_{i}$ when we pass from $i=n-1$ to $i=0$. Hence $\tau\left(M_{n}\right) \geq n$ if $n \geq 4$, and $M_{n}$ is not eigensharp if $n=3 k$ and $k \geq 2$. Finally, $M_{3}$ is a 6-cycle with three crossing chords, i.e. $M_{3}=K_{3,3}$, so $\tau\left(M_{3}\right)=r\left(M_{3}\right)=1$.

Finally, consider the cartesian product $C_{m} \square C_{n}$, also called a discrete torus. Some discrete tori are eigensharp. The exact computation of $\tau(G)$ is a delicate question, which will be considered in a subsequent paper due to the detailed combinatorial nature of the optimal decompositions. For the present, we discuss eigensharpness in some simple cases that do not require complex combinatorial arguments. Most of our effort in the remainder of this section will be devoted to determining the signature of $C_{m} \square C_{n}$, which we denote $\left(p_{m, n}, s_{m, n}, q_{m, n}\right)$.

LEMMA 1. Let $I_{k}$ denote the multiset of the first $k$ terms in the sequence $(0,1,1,2,2, \ldots)$. Then $p_{m, n}, s_{m, n}, q_{m, n}$ are, respectively, the number of positive, zero, and negative elements in the multiset $\left\{\frac{1}{2}-(i / m+j / n): i \in I_{m}, j \in I_{n}\right\}$. In particular, if $d$ denotes the greatest common divisor of $m$ and $n$, then $s_{m, n}=$ $2 d-2,0,2 d-1$, respectively, as $m, n$ are both even, both odd, or of opposite parity.

PrOOF. Let $A_{m, n}$ denote the full collection of eigenvalues of $C_{m} \square C_{n}$; it follows from the discussion of eigenvalues in the introduction that $A_{m, n}$ is the multiset $\{2 \cos (2 \pi i / m)+2 \cos (2 \pi j / n): 0 \leq i \leq m-1,0 \leq j \leq n-1\}$. Since $\cos 2 \pi \alpha=$ $\cos 2 \pi(1-\alpha)$, we can replace the index set by $i \in I_{m}, j \in I_{n}$. We may also apply the trigonometric identity $\cos \alpha+\cos \beta=2 \cos [(\alpha+\beta) / 2] \cos [(\alpha-\beta) / 2]$ to write

$$
A_{m, n}=\left\{4 \cos \left(\frac{i}{m}+\frac{j}{n}\right) \pi \cos \left(\frac{i}{m}-\frac{j}{n}\right) \pi: i \in I_{m}, j \in I_{n}\right\} .
$$

Note that $i \in I_{k}$ implies $i \leq k / 2$, with equality possible if and only if $k$ is even. Hence $|i / m-j / n|<\frac{1}{2}$ when $i \in I_{m}, j \in I_{n}$ (unless $(i, j)=(0, n / 2)$ or $\left.(m / 2,0)\right)$, so the sign of the corresponding eigenvalue is determined by the sign of the first factor, which is $+, 0,-$ according to whether $\frac{1}{2}-(i / m+j / n)$ is $+, 0,-$. In the exceptional case, $i / m+j / n=\frac{1}{2}$ and the same criterion holds. 
Hence $s_{m, n}$ is the number of solutions to $i n+j m=m n / 2$ with $i \in I_{m}, j \in I_{n}$. Define $m^{\prime}, n^{\prime}$ by $m=d m^{\prime}, n=d n^{\prime}$. We need to count solutions to $i n^{\prime}+j m^{\prime}=$ $d m^{\prime} n^{\prime} / 2$. When $m, n$ are both odd, $d m^{\prime} n^{\prime} / 2$ is not an integer and there are no solutions.

Suppose $m, n$ are both even, so $d=2 d^{\prime}$ is even. Since $m^{\prime}, n^{\prime}$ are relatively prime, solutions require $m^{\prime} \mid i$ and $n^{\prime} \mid j$. Hence for each solution we can write $i=a m^{\prime}$ and $j=b n^{\prime}$ with $0 \leq a, b \leq d^{\prime}$ and $a+b=d^{\prime}$. Due to the multiplicity of $b=d^{\prime}-a$ in $I_{d}$, for each choice of $a \in I_{d}$ we have two (equal) suitable choices of $j \in I_{n}$, except that when $a=0$ or $a=d^{\prime}$ we have only one. Since $a=0$ and $a=d^{\prime}$ appear once in $I_{d}$ we have a total of $2 d-2$ solution pairs $i \in I_{m}, j \in I_{n}$.

Suppose $m, n$ have opposite parity, with $m$ even. Then $d$ is odd and $m^{\prime}=2 m$ is even, and we can rewrite the equation as $i n^{\prime}+2 j m=d m n^{\prime}$. As before, solutions require $m \mid i$ and $n^{\prime} \mid j$. Hence we can write $i=a m$ and $j=b n^{\prime}$ with $0 \leq a \leq d$, $0 \leq b \leq(d-1) / 2$, and $a+2 b=d$. Due to the multiplicity of $a=d-2 b$ in $I_{2 d}$, for each choice of $b \in I_{d}$ we have two (equal) suitable choices of $i \in I_{m}$, except only one when $b=0$. Since $b=0$ appears once in $I_{d}$ we have a total of $2 d-1$ solution pairs $i \in I_{m}, j \in I_{n}$.

In order to compute the signature of a graph on a given number of vertices, it suffices to know $s(G)$ and $p(G)$. Hence we complete the determination of the signatures of discrete tori by computing $p_{m, n}$.

LEMMA 2. In the case that $m, n$ are (1) both even, (2) both odd, or (3) of opposite parity, respectively, $p_{m, n}$ is given by

(1) $p_{m, n}=m n / 2-d+1$.

(2) $p_{m, n}=m+n-1+4 \sum_{k=1}^{(m-1) / 2}\lfloor n(2 k-1) / 2 m\rfloor$.

(3) Either of the expressions below, where $m$ is even.

(3a) $p_{m, n}=m+n-2 d+4 \sum_{k=1}^{m / 2-1}\lfloor n k / m\rfloor$.

(3b) $p_{m, n}=m+n-2 d+4 \sum_{k=1}^{(n-1) / 2}\lfloor m(2 k-1) / 2 n\rfloor$.

ProOF. Case 1. Since $C_{m} \square C_{n}$ is bipartite, $p_{m, n}=q_{m, n}$. Hence $p_{m, n}=$ $\left(m n-s_{m, n}\right) / 2=m n / 2-d+1$, by Lemma 1 .

Case 2. We want to count pairs $i \in I_{m}, j \in I_{n}$ with $i / m+j / n<\frac{1}{2}$. This always holds if $i=0$ or $j=0$, which contributes $m+n-1$ positive eigenvalues. Otherwise, with $1 \leq i \leq(m-1) / 2$ and $1 \leq j \leq(n-1) / 2$ each pair $(i, j)$ appears four times. Consider a particular $i$. Since $s_{m, n}=0$ by Lemma $1, i / m+j / n<\frac{1}{2}$ is equivalent to $j \leq n / 2-i n / m=n(m-2 i) / 2 m$. Letting $k=(m+1) / 2-i$ and summing over the nonzero options for $i$ gives the formula (2). Of course, we can reverse the roles of $m$ and $n$ in this formula.

Case 3. The analysis is similar to the previous case, with two minor complications: the structure of $I_{m}$ and the existence of solutions to $i / m+j / n=\frac{1}{2}$. Again we have $i / m+j / n<\frac{1}{2}$ whenever $i=0$ or $j=0$ unless $(i, j)=(m / 2,0)$, which contributes $m+n-2$ positive eigenvalues. When $i=m / 2$, we get one 0 and $n-1$ negative eigenvalues. We have considered all $i \in I_{m}, j \in I_{n}$ that appear singly; the remaining eigenvalues come in groups of four. To count the values $(i, j)$ that yield $i / m+j / n \leq \frac{1}{2}$, we require for each $j$ that $j \leq n(m / 2-i) / m$. Letting $k=m / 2-i$ and summing over the nonzero options for $i$ gives $p_{m, n}+s_{m, n}=$ $m+n-2+1+4 \sum_{i=1}^{m / 2-1}\lfloor i n / m\rfloor$. Subtracting the value $s_{m, n}=2 d-1$ given by 
Lemma 1 yields (3a). Using the same approach with the inequality $i \leq m(n-2 j) / 2 n$ and letting $k=(n+1) / 2-i$ yields $(3 \mathrm{~b})$.

The discussion of equality in the next lemma gives a glimpse of the combinatorial arguments needed to characterize optimal decompositions of $C_{m} \square C_{n}$.

LEMMA 3. $\tau\left(C_{m} \square C_{n}\right) \geq m n / 2$, with equality if and only if $m$ and $n$ are even.

PROOF. The only complete bipartite subgraphs in $C_{m} \square C_{n}$ are 4-cycles and stars with at most 4 edges. Since $C_{m} \square C_{n}$ has $2 m n$ edges, $\tau\left(C_{m} \square C_{n}\right) \geq m n / 2$. We refer to the cycle $\{(i, j),(i, j+1),(i+1, j+1),(i+1, j)\}$ as the 4-cycle at $(i, j)$. If $m, n$ are even, we can achieve $\tau\left(C_{m} \square C_{n}\right)=m n / 2$ by stars at $\{(i, j): i, j$ are even\}, or by a checkerboard of cycles at the same set, or by various intermediate configurations.

Conversely, suppose equality is achieved by some decomposition $D$. Then every subgraph in $D$ covers exactly 4 edges, and each edge of $C_{m} \square C_{n}$ belongs to exactly one subgraph in $D$. In particular, there are no adjacent centers of stars, nor a star centered on a vertex of a 4-cycle in $D$. Let $S$ be the set of vertices that are centers of stars in $D$.

Suppose $n$ is odd. Consider a row of $n$ vertices in $C_{m} \square C_{n}$, and consider the cycle $C$ formed by these vertices. If the edges of $C$ all belong to 4 -cycles in $D$, then the cycles alternate being above and below the row, and $n$ is even. Hence every row contains a vertex of $S$. If the distance between (cyclically) successive vertices of $S$ in a row is always even, then $n$ is even. Hence every row has a pair of vertices in $S$ at an odd distance $2 k+1$ such that all $2 k-1$ edges between them other than the incident edges belong to 4-cycles in $D$.

From all pairs of horizontally successive vertices of $S$, choose the pair with the smallest odd distance, $2 k+1$. Let these be $(i, j)$ and $\left(i, j^{\prime}\right)$, with $j^{\prime}-j=2 k+1$. Since we cannot have adjacent centers of stars, $k>0$. However, when $k>0$ we can find a closer pair. The edge from $\left(i, j^{\prime}-1\right)$ to $\left(i, j^{\prime}\right)$ belongs to the star at $\left(i, j^{\prime}\right)$, but the edge from $\left(i, j^{\prime}-2\right)$ to $\left(i, j^{\prime}-1\right)$ must belong to a cycle, since we chose a closest pair. By reflective symmetry, we may assume this is the cycle at $\left(i, j^{\prime}-2\right)$, which is above row $i$. Proceeding back toward $(i, j)$, the cycles must alternate being above and below row $i$, so that the cycle containing the edge from $(i, j+1)$ to $(i, j+2)$ is the cycle at $(i, j+1)$, which lies above row $i$ (see Figure 1 -in these illustrations, the large dots represent centers of stars and the inscribed circles represent 4 -cycles in the decompositions).

We now claim that $(i-1, j+1),\left(i-1, j^{\prime}-1\right) \in S$. Consider the edges from these vertices to $(i, j+1)$ and $\left(i, j^{\prime}-1\right)$. The cycles at $(i, j+1),\left(i, j^{\prime}-2\right)$ and stars at $(i, j),\left(i, j^{\prime}\right)$ guarantee that these edges can only be covered by stars at $(i-1, j+1)$, $\left(i-1, j^{\prime}-1\right)$. However, this gives horizontally successive stars at distance $2 k-1$, contradicting the minimality of the initial choice. Hence $n$ is even, and by the same reasoning $m$ is also even.

These elementary ideas suffice to decide eigensharpness in some cases.

THEOREM 6. Eigensharpness of discrete tori.

(1) If $m$ and $n$ are even, $C_{m} \square C_{n}$ is not eigensharp.

(2) If $n$ is odd and $m=2$ tn with $t \leq n, C_{m} \square C_{n}$ is not eigensharp.

(3) If $n$ is odd and $m=(2 t+1) n$, then $C_{m} \square C_{n}$ is eigensharp. 


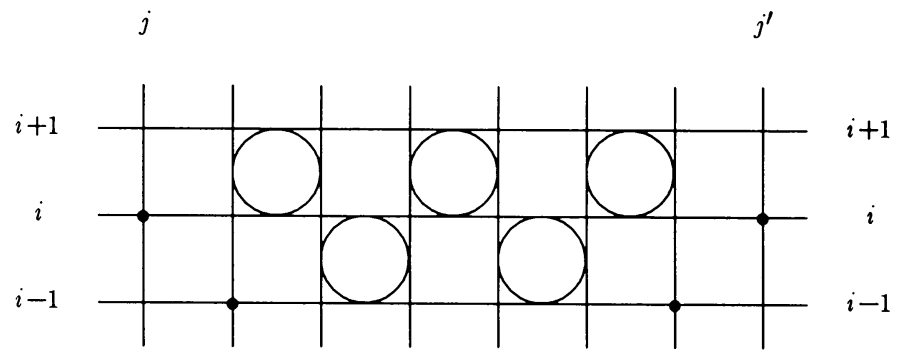

FIGURE 1. Propagation of stars

ProOF. (1) By the lemmas, $p_{m, n}=m n / 2-d+1 \leq m n / 2-1<m n / 2=$ $\tau\left(C_{m} \square C_{n}\right)$.

(2) With $m=2 t n$, we have $d=n$, so Lemma 2.2 reduces to $p_{m, n}=2 t n+n-2 n+$ $4 \sum_{k=1}^{(n-1) / 2}\lfloor t(2 k-1)\rfloor=t n^{2}+t-n$. Also $s_{m, n}=2 n$, and so $q_{m, n}=t n^{2}-t-n$. Hence $r\left(C_{m} \square C_{n}\right)=t n^{2}+t-n \leq t n^{2}=m n / 2$. Since $m, n$ are odd, Lemma 3 forbids eigensharpness.

(3) With $m=(2 t+1) n$, again $d=n$. The summand in Lemma 2.2 reduces to $\lfloor(2 t+1)(2 k-1) / 2\rfloor=2 k t+k-t-1$. The full sum becomes $p_{m, n}=(2 t+2) n-$ $1+4 \sum_{k=1}^{(n-1) / 2}(2 k t+k-t-1)=(2 t+1)\left(n^{2}+1\right) / 2$, by a routine calculation. Since $s_{m, n}=0, q_{m, n}=(2 t+1)\left(n^{2}-1\right) / 2$. Hence $r\left(C_{m} \square C_{n}\right)=p_{m, n}$ and we need to construct a decomposition of this size. First consider $C_{n} \square C_{n}$; it can be decomposed into $((n-1) / 2)^{2}$ 4-cycles and $((n+1) / 2)^{2}$ stars, with the stars and cycles each arranged in "diamonds" as indicated in Figure 2; this totals $\left(n^{2}+1\right) / 2$, as desired. In general, this pattern can be repeated $2 t+1$ times, with each repetition adding $\left(n^{2}+1\right) / 2$ subgraphs, as illustrated in Figure 3 .

Additional results about eigensharpness of the discrete torus require more detailed consideration of the optimal configurations. For example, $C_{m} \square C_{n}$ is eigensharp if $m=n+2 k$ and $n \equiv 1$ or $3(\bmod 4 k)$, and $C_{m} \square C_{n}$ is not eigensharp if $m=k n \pm 1$. These results will appear in a subsequent paper.
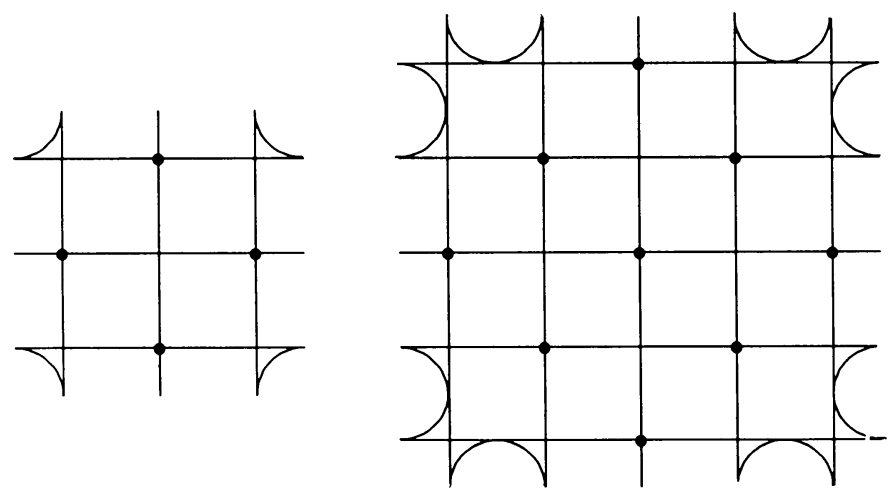

FIGURE 2. Optimal decomposition of $C_{3} \square C_{3}$ and $C_{5} \square C_{5}$ 


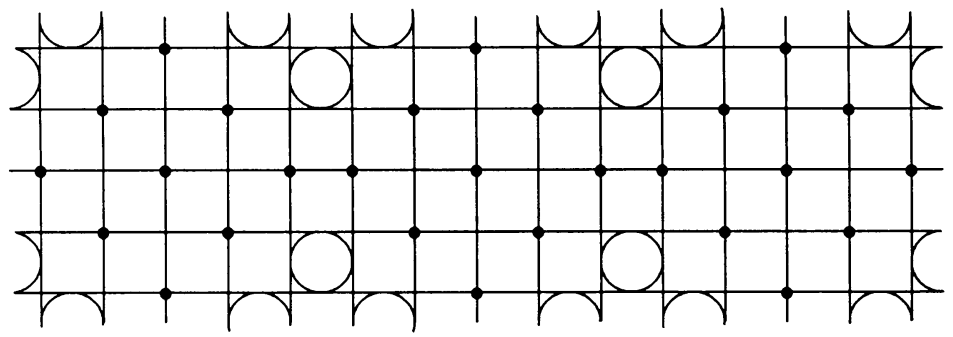

FIGURE 3. Optimal decomposition of $C_{5} \square C_{15}$

4. Weak products. Now we consider graphs that can be expressed as weak products. Note that the rule for computing eigenvalues of the weak product yields $p(G * H)=p(G) p(H)+q(G) q(H)$ and $q(G * H)=p(G) q(H)+q(G) p(H)$. A simple construction gives a decomposition of the weak product that is sometimes optimal.

LEMMA 4. For any graphs $G, H, \tau(G * H) \leq 2 \tau(G) \tau(H)$.

PROOF. Given optimal decompositions of the factors into $\tau(G)$ and $\tau(H)$ complete bipartite subgraphs, we need only exhibit a decomposition of $G * H$ into $2 \tau(G) \tau(H)$ complete bipartite subgraphs. With every choice of an edge in $G$ given by $u \leftrightarrow v$ and an edge in $H$ given by $x \leftrightarrow y$, we associate two edges in $G * H$, namely $(u, x) \leftrightarrow(v, y)$ and $(u, y) \leftrightarrow(v, x)$. Every edge of $G * H$ belongs to a unique pair of this type. If $K_{U, V}$ and $K_{X, Y}$ are subgraphs in respective optimal decompositions of $G, H$, we form two complete bipartite subgraphs of $G * H$ to account for all edge pairs in $G * H$ arising from these edges. The two subgraphs are $K_{U \times X, V \times Y}$ and $K_{U \times Y, V \times X}$. The subgraph pairs contain all pairs of an edge from $G$ and an edge from $H$, hence we get all edge-pairs in $G * H$. More formally, if $G=\bigcup K_{U_{i}, V_{i}}$ and $H=\bigcup_{X_{j}, Y_{j}}$, then we have shown $G * H=\bigcup\left(K_{U_{i} \times X_{j}, V_{i} \times Y_{j}} \cup K_{U_{i} \times Y_{j}, V_{i} \times X_{j}}\right)$.

Let $\mathbf{B}$ denote the class of eigensharp graphs with $p(G)=q(G)$; note that $\mathbf{B}$ includes all eigensharp bipartite graphs, such as trees, complete bipartite graphs, and $C_{n}$ with $n \equiv 2 \bmod 4$.

THEOREM 7. The class $\mathbf{B}$ is closed under finite weak products.

PrOOF. It suffices to consider a pair $G, H$, since the weak product is associative. Since $p(G)=q(G)$ and $p(H)=q(H)$, we have $p(G * H)=q(G * H)=2 r(G) r(H)$. This in turn equals $2 \tau(G) \tau(H)$ since $G, H$ are eigensharp. Lemma 4 gives a decomposition of this size, so $G * H$ is also eigensharp.

Sometimes, we can get eigensharp graphs even when the factors are not both eigensharp.

THEOREM 8. If $G \in \mathbf{B}$ and $H$ has no zero eigenvalues, then $G * H \in \mathbf{B}$.

ProOF. Computation yields $p(G * H)=q(G * H)=p(G) \cdot|V(H)|$, so we need only show $\tau(G * H)=p(G) \cdot|V(H)|$. For each $K_{X, Y}$ in an optimal decomposition of $G$ and each $v \in V(H)$, use the subgraph $K_{X \times\{v\}, Y \times \operatorname{Adj}(v)}$ of $G * H$. This yields a decomposition of the desired size.

In [9] we decomposed finite weak products of complete graphs into the number of complete bipartite subgraphs given by the eigenvalue bound. To generalize this result, we define the class $\mathbf{H}$ mentioned in the introduction, i.e. the eigensharp 
star-coverable graphs with no zero eigenvalues. This class includes the complete graphs and cycles of length $\neq 4 k$. We next show that weak products of graphs in $\mathbf{H}$ are always eigensharp, though they need not belong to $\mathbf{H}$. This shows that the statement of [9] that $C_{5} * C_{5}$ is not eigensharp is false. We also note that $C_{n} \square C_{n}=C_{n} * C_{n}$ when $n$ is odd, via the isomorphism sending $(i, j) \in V\left(C_{n} \square C_{n}\right)$ to $(i+j, i-j) \in V\left(C_{n} * C_{n}\right)$, where the vertices of each are $\{0, \ldots, n-1\} \times$ $\{0, \ldots, n-1\}$ and all addition is taken modulo $n$. Hence this theorem yields an immediate proof that $C_{n} \square C_{n}$ is eigensharp when $n$ is odd. The fact that weak products of graphs in $\mathbf{H}$ need not belong to $\mathbf{H}$ (for example, $C_{3} * C_{3}$ is not starcoverable) means that this theorem cannot be proved by induction on the number of graphs in the product.

\section{THEOREM 9. Any finite weak product of graphs in $\mathbf{H}$ is eigensharp.}

PrOOF. Let $G_{1}, \ldots, G_{k}$ be the factors and $G$ the weak product. The vertices of $G$ are $k$-tuples in which the $i$ th coordinate is a vertex of $G_{i}$. Given a fixed decomposition of each $G_{i}$ into $\tau\left(G_{i}\right)$ stars, a vertex of $G_{i}$ that is the center of a star in this decomposition is called a $h u b$ of $G_{i}$; the other vertices are nonhubs.

We begin by computing the eigenvalue bound $r(G)$. Let $p_{i}=p\left(G_{i}\right), q_{i}=q\left(G_{i}\right)$, $n_{i}=\left|V\left(G_{i}\right)\right|, c_{i}=\max \left\{p_{i}, q_{i}\right\}, d_{i}=\min \left\{p_{i}, q_{i}\right\}$. Note that $p_{i}+q_{i}=c_{i}+d_{i}=n_{i}$, since $G_{i} \in \mathbf{H}$. The formulas for $p(G * H)$ and $q(G * H)$ generalize to multiple products as

$$
p(G)=\sum_{|S| \text { even }}\left(\prod_{i \notin S} p_{i}\right)\left(\prod_{i \in S} q_{i}\right) \text { and } q(G)=\sum_{|S| \text { odd }}\left(\prod_{i \notin S} p_{i}\right)\left(\prod_{i \in S} q_{i}\right)
$$

where the sums are over all subsets $S \subseteq\{1, \ldots, k\}$. If we count up (in two ways) all products of an eigenvalue from each $G_{i}$, weighting the positive products with +1 and the negative products with -1 , we get $p(G)-q(G)=\prod_{i}\left(p_{i}-q_{i}\right)$, since we get a positive product precisely when choosing an even number of negative eigenvalues. If we interchange $p_{i}$ and $q_{i}$, we maintain the sum $p(G)+q(G)=\prod_{i} n_{i}=\prod_{i}\left(p_{i}+q_{i}\right)$ and change the sign of the difference, thus preserving the set $\{p(G), q(G)\}$. Thus if we replace each $\left(p_{i}, q_{i}\right)$ by $\left(c_{i}, d_{i}\right)$ and note that $\prod_{i}\left(c_{i}-d_{i}\right) \geq 0$ by construction, we conclude that $r(G)=\sum_{|S| \text { even }}\left(\prod_{i \notin S} c_{i}\right)\left(\prod_{i \in S} d_{i}\right)$.

Now we must decompose $G$ into $\sum_{|S| \text { even }}\left(\prod_{i \notin S} c_{i}\right)\left(\prod_{i \in S} d_{i}\right)$ complete bipartite subgraphs. We will first specify the subgraphs, and later show that each edge appears in exactly one of these subgraphs. For an arbitrary edge $(\bar{u}, \bar{v})$ in $G$, we have $u_{i} \leftrightarrow v_{i}$ in $G_{i}$ for all $i$. Since by hypothesis the edges of $G_{i}$ are covered by $c_{i}$ stars on hubs, at least one of $\left\{u_{i}, v_{i}\right\}$ is a hub in $G_{i}$.

We need to define $\prod_{i \notin S} c_{i} \prod_{i \in S} d_{i}$ subgraphs for each $S$ of even size. For $S=\varnothing$ this is $\prod_{i \notin S} c_{i}$, which is the number of ways to choose a hub in each coordinate. Each such choice specifies a vertex in $G$, and we use a star centered at that vertex as the corresponding subgraph. In general, the star includes all edges incident to that vertex, but when there is an edge joining two such vertices we arbitrarily choose to include it in only one of the two stars. In any case, these stars partition all edges incident to vertices composed entirely of hubs.

The remaining edges have at least one nonhub among the coordinates of each endpoint. In general, $\prod_{i \notin S} c_{i} \prod_{i \in S} d_{i}$ is the number of ways to choose a single hub from each $G_{i}$ with $i \notin S$ and a single nonhub from each $G_{i}$ with $i \in S$. From 
each such sequence of choices $\bar{f}=\left(f_{1}, \ldots, f_{k}\right)$, we must define a unique subgraph $K_{U, V}=K(\bar{f})$. We call $\bar{f}$ the sequence of fixed values for this subgraph. For example, if $k=11$ and $S=\{3,5,7,8,9,11\}$, we may choose

$$
\bar{f}=\left(h_{j_{1}}^{1}, h_{j_{2}}^{2}, x_{j_{3}}^{3}, h_{j_{4}}^{4}, x_{j_{5}}^{5}, h_{j_{6}}^{6}, x_{j_{7}}^{7}, x_{j_{8}}^{8}, x_{j_{9}}^{9}, h_{j_{10}}^{10}, x_{j_{11}}^{11}\right),
$$

where $h_{j}^{i}$ denotes the $j$ th hub vertex of $G_{i}$ and $x_{j}^{i}$ denotes the $j$ th nonhub vertex of $G_{i}$. We define the corresponding subgraph $K_{U, V}=K(\bar{f})$ by making each partite set a cartesian product in which the $i$ th factor is either $\left\{f_{i}\right\}$ or $\operatorname{Adj}\left(f_{i}\right)$. It remains to specify which partite set gets the factor $\left\{f_{i}\right\}$ for each $i$.

To complete this task, view the coordinates cyclically, and write $f_{i}$ for the 1element set $\left\{f_{i}\right\}$. Among the coordinates in $S$, let $f_{i}$ alternate between $U$ and $V$ as a factor. For coordinates not in $S$, assign the fixed factor $f_{i}$ to the same set that has the fixed factor in the next higher coordinate that belongs to $S$. For the example $\bar{f}$ chosen above, $K(\bar{f})$ has the partite sets given below, where we have written $X_{j}^{i}, H_{j}^{i}$ for $\operatorname{Adj}\left(x_{j}^{i}\right), \operatorname{Adj}\left(h_{j}^{i}\right)$.

$$
\begin{aligned}
& U=H_{j_{1}}^{1} \times H_{j_{2}}^{2} \times X_{j_{3}}^{3} \times h_{j_{4}}^{4} \times x_{j_{5}}^{5} \times H_{j_{6}}^{6} \times X_{j_{7}}^{7} \times x_{j_{8}}^{8} \times X_{j_{9}}^{9} \times h_{j_{10}}^{10} \times x_{j_{11}}^{11}, \\
& V=h_{j_{1}}^{1} \times h_{j_{2}}^{2} \times x_{j_{3}}^{3} \times H_{j_{4}}^{4} \times X_{j_{5}}^{5} \times h_{j_{6}}^{6} \times x_{j_{7}}^{7} \times H_{j_{8}}^{8} \times x_{j_{9}}^{9} \times H_{j_{10}}^{10} \times X_{j_{11}}^{11} .
\end{aligned}
$$

Note that, because $S$ has even size, interchanging $U$ and $V$ yields the same subgraph. Also, this description holds for the stars defined when $S=\varnothing$, except that then we sometimes do not use all of $\operatorname{Adj}\left(f_{i}\right)$.

Showing that each edge of $G$ appears in exactly one of the subgraphs will prove that this is a decomposition. We need only consider edges with at least one nonhub in each endpoint, since the others are supplied by the stars. To show there is a unique subgraph containing the edge between $\bar{u}$ and $\bar{v}$, it suffices to show there is a unique choice of the fixed values determining a subgraph containing it. For example, consider the edge $e^{*}$ between

$$
\bar{u}=\left(x_{1}^{1}, h_{3}^{2}, h_{1}^{3}, h_{1}^{4}, x_{2}^{5}, x_{2}^{6}, h_{4}^{7}, x_{3}^{8}, h_{2}^{9}, h_{1}^{10}, x_{5}^{11}\right)
$$

and

$$
\bar{v}=\left(h_{2}^{1}, h_{2}^{2}, x_{1}^{3}, h_{2}^{4}, h_{2}^{5}, h_{2}^{6}, x_{3}^{7}, h_{3}^{8}, x_{1}^{9}, x_{1}^{10}, h_{1}^{11}\right) .
$$

To recover $\bar{f}$, we begin by recovering the set $S$ of coordinates in which $\bar{f}$ is a nonhub. This must be a subset of the coordinates in which nonhub values appear, which in our example are all but $i=2,4$. Note that in each coordinate at most one of $\bar{u}, \bar{v}$ has a nonhub, because $G_{i}$ is star-coverable. We claim $S$ for the edge $\overline{u v}$ is determined by the following rule: if $u_{i}$ or $v_{i}$ is a nonhub, then $i \in S$ if and only if the next lower coordinate having a nonhub value has it in the other vertex. To prove this, we may assume by symmetry that $u_{i}$ is a nonhub and $v_{i}$ is a hub, and suppose $j$ is the next lower coordinate with a nonhub. If $i \in S$, then $f_{i}=u_{i}$. If also $j \in S$, then $f_{j}$ is a nonhub and by construction $v_{j}=f_{j}$. If $j \notin S$, then $f_{j}$ is a hub and appears as $u_{j}$, again making $v_{j}$ the nonhub. Now suppose $i \notin S$, so $f_{i}$ is a hub, i.e. $f_{j}=v_{j}$. By construction the next-higher coordinate in $S$ has its nonhub in $\bar{v}$. If $j \in S$, then $u_{j}=f_{j}$ is a nonhub, while $j \notin S$ makes $f_{j}$ a hub on the same side as $f_{i}$, so again $u_{j}$ is the nonhub. Thus the edge $e^{*}$ above can only come from a graph with $S=\{3,4,7,8,9,11\}$. (Note: [9] contains a misprint in the statement of the rule for retrieving $S$, where "next" should read "next lower".) 
Having determined $S$, it is easy to retrieve $\bar{f}$. The nonhubs are the nonhubs in coordinates belonging to $S$, and for the remaining coordinates we select the hub on the same side as the nonhub in the next higher coordinate in $S$. Thus the edge $e^{*}$ is an edge only in the subgraph determined by the fixed values $\left(h_{2}^{1}, h_{2}^{2}, x_{1}^{3}, h_{1}^{4}, x_{2}^{5}, h_{2}^{6}, x_{3}^{7}, x_{3}^{8}, x_{1}^{9}, h_{1}^{10}, x_{5}^{11}\right)$, which belongs to the class of graphs described above for $S=\{3,4,7,8,9,11\}$.

The argument of the preceding theorem can be extended to a slightly more general class. In the proof, the lack of zero eigenvalues gave us the fact that the number of subgraphs desired from the set $S$ equalled the number of ways to choose nonhubs from $S$ and hubs from the remaining coordinates. With appropriate generalizations of the concepts of hub and nonhub, we can maintain this property and strengthen the theorem by weakening the conditions on $\mathbf{H}$. This also leads us to an example of eigensharp graphs whose weak product is not eigensharp. The construction we form for the product generalizes that of the previous theorem. However, the fact that this cannot be extended to weak products of arbitrary eigensharp graphs makes the next theorem of mostly technical interest, and therefore we presented the more intuitive special case separately above.

In the proof of Theorem 8 we used the stars at each vertex of one factor. In the proof of Theorem 9 we again used stars at every vertex in each factor, although some were hubs and some were nonhubs. An important property of the collection of all stars in a graph is that it covers the edges exactly twice each. Surprisingly, it is important to maintain this property when generalizing the idea of star-coverability.

A hub/rim decomposition of order $(c, d)$ is a collection of $c+d$ complete bipartite subgraphs together covering each edge of a graph exactly twice and satisfying the following two properties. (1) The first $c$ subgraphs $K_{H_{i}, I_{i}}$ correspond to disjoint sets of vertices $\left\{H_{i}\right\}$ called hubs. (2) The remaining $d$ subgraphs $K_{R_{i}, S_{i}}$ partition the edges between the vertices not in hubs and the vertices in hubs, with the sets $R_{i}$ of nonhub vertices called rims.

Several comments about hub/rim decompositions are immediate. The rims need not be disjoint, though the edges of the rim subgraphs are disjoint. The vertices that do not belong to hubs form an independent set. In each $K_{R_{i}, S_{i}}, S_{i} \subseteq \bigcup_{j} H_{j}$. In the $K_{H_{i}, I_{i}}$, there is no restriction on $I_{i}$. Star-coverable graphs have hub/rim decompositions of order $(\tau(G), n-\tau(G))$, with all rims being single vertices.

LEMMA 5. A graph with a hub/rim decomposition of order $(c, d)$ has a decomposition into $c$ complete bipartite subgraphs. Furthermore, the edges between two hubs $H_{i}$ and $H_{j}$ form the complete bipartite subgraph $K_{H_{i} \cap I_{j}, H_{j} \cap I_{i}}$.

PROOF. We obtain the decomposition from the hub subgraphs. Because the rim subgraphs partition the edges between hubs and nonhubs, the hub subgraphs also cover them once each. The hub subgraphs doubly cover the edges between hubs; we must trim them to avoid this. An edge between $H_{i}$ and $H_{j}$ is doubly covered if and only if it is covered in the $i$ th and $j$ th hub subgraphs, i.e. the endpoint in $H_{j}$ belongs to $I_{i}$ and the endpoint in $H_{i}$ belongs to $I_{j}$. Hence $K_{H_{i} \cap I_{j}, H_{j} \cap I_{i}}$ is the subgraph of duplicated edges. To correct this for each $i, j$, simply delete from each $I_{i}$ all hub vertices belonging to any $H_{j}$ with $j>i$. This is consistent because the hubs are disjoint. The result is still a decomposition, because deleting these vertices removes no edges from hubs to nonhubs. 
Let $\mathbf{J}$ be the class of graphs with hub/rim decompositions of order $(\max \{p(G), q(G)\}, \min \{p(G), q(G)\}) ;$ since $\tau(G) \leq c$ as noted above, graphs in $\mathbf{J}$ are eigensharp. Eigensharp star-coverable graphs with no zero eigenvalues (i.e., graphs in $\mathbf{H}$ ) belong to $\mathbf{J}$; for each zero eigenvalue, the edges involving nonhubs must be collapsed into one less rim graph to remain in $\mathbf{J}$. The smallest graph in $\mathbf{J}$ with a zero eigenvalue is $G=K_{4}-e$, with $V(G)=\{u, v, x, y\}$ and $E(\bar{G})=\{(x, y)\}$. The signature of $G$ is $(1,1,2)$. $G$ actually has two hub/rim decompositions of order $(2,1)$, described as follows. (1) $G$ is star-coverable, with $u, v$ as 1-vertex hubs. The single rim graph is then $K_{\{u, v\},\{x, y\}}$. (2) $G$ is also coverable by a 4-cycle and a single star. Now $\{x, y\},\{u\}$ are the hubs, with $K_{\{x, y\},\{u, v\}}, K_{\{u\},\{x, y\}}$ the hub graphs and $K_{\{v\},\{x, y\}}$ the rim graph. In considering products, we fix a single representation by hubs and rims.

THEOREM 10. Any finite weak product of graphs with hub/rim decompositions of orders $\left(c_{i}, d_{i}\right)$ has a decomposition into $\sum_{|S| \text { even }}\left(\prod_{i \notin S} c_{i}\right)\left(\prod_{i \in S} d_{i}\right)$ complete bipartite subgraphs. In particular, any weak product of graphs in $\mathbf{J}$ is eigensharp.

PROOF. For graphs in $\mathbf{J}$, the previous computation for the eigenvalue bound for the weak product is valid, so we need only construct and verify the decomposition into $\sum_{|S| \text { even }}\left(\prod_{i \notin S} c_{i}\right)\left(\prod_{i \in S} d_{i}\right)$ complete bipartite subgraphs. The construction generalizes that for the previous theorem, and the verification is very similar. Fix hub/rim decompositions with $c_{i}$ hubs $H_{j}^{i}$ and $d_{i}$ rims $R_{j}^{i}$ for $G_{i}$, letting the hub subgraphs be $K_{H_{i}^{j}, I_{i}^{j}}$ and the rim subgraphs be $K_{R_{i}^{j}, S_{i}^{j}}$. Again $\prod_{i \notin S} c_{i} \prod_{i \in S} d_{i}$ is the number of ways to choose a hub from each $G_{i}$ with $i \notin S$ and a rim from each $G_{i}$ with $i \in S$. Such a choice is the sequence of fixed sets $\bar{f}=\left(f_{1}, \ldots, f_{k}\right)$ for the subgraph to be constructed. Again we construct $K_{U, V}=K(\bar{f})$ by letting $U, V$ be cartesian products. If $f_{i}=H_{j}^{i}$, then the $i$ th factor is $H_{j}^{i}$ in one of them and $I_{j}^{i}$ in the other. If $f_{i}=R_{j}^{i}$, then the $i$ th factor is $R_{j}^{i}$ in one of them and $S_{j}^{i}$ in the other. The rule for distributing the fixed sets is cyclic as before, so that if $k=11$ and $S=\{3,5,7,8,9,11\}$ the resulting subgraph looks like

$$
\begin{aligned}
& U=I_{j_{1}}^{1} \times I_{j_{2}}^{2} \times S_{j_{3}}^{3} \times H_{j_{4}}^{4} \times R_{j_{5}}^{5} \times I_{j_{6}}^{6} \times S_{j_{7}}^{7} \times R_{j_{8}}^{8} \times S_{j_{9}}^{9} \times H_{j_{10}}^{10} \times R_{j_{11}}^{11}, \\
& V=H_{j_{1}}^{1} \times H_{j_{2}}^{2} \times R_{j_{3}}^{3} \times I_{j_{4}}^{4} \times S_{j_{5}}^{5} \times H_{j_{6}}^{6} \times R_{j_{7}}^{7} \times I_{j_{8}}^{8} \times R_{j_{9}}^{9} \times I_{j_{10}}^{10} \times S_{j_{11}}^{11} .
\end{aligned}
$$

As in the previous proof, an edge between vertices whose coordinates all belong to hubs appears twice in the graphs generated by $S=\varnothing$. We correct this doublecovering in the same manner as done for the decomposition in Lemma 5 . Consider a graph $K_{U, V}$ generated by $S=\varnothing$, with $U=H_{j_{1}}^{1} \times \cdots \times H_{j_{k}}^{k}$ and $V=I_{j_{1}}^{1} \times \cdots \times I_{j_{k}}^{k}$. Delete from the $i$ th factor $I_{j_{i}}^{i}$ of $V$ all vertices belonging to hubs $H_{j^{\prime}}^{i}$ in $G_{i}$ with $j^{\prime}>j_{i}$.

Again we must consider edges between vertices with at least one nonhub to verify the decomposition. Consider the edge $(\bar{u}, \bar{v})$ as before; we retrieve the subgraph containing it. In each coordinate at most one of $\bar{u}, \bar{v}$ has a nonhub. The rule for recovering $S$ is the same as before: if $u_{i}$ or $v_{i}$ is a nonhub, then $i \in S$ if and only if the next lower coordinate having a nonhub value has it in the other vertex. The proof of the rule is also unchanged.

There is one additional wrinkle in determining the fixed sets, because the rims partition not the nonhub vertices, but rather their incident edges. However, this is 
easily handled; if $i \in S$, then the hub vertex in coordinate $i$ opposite the nonhub vertex determines which rim was used to obtain this factor edge; this rim is $f_{i}$. Since the hubs are disjoint in $G_{i}$, the choice of $f_{i}$ in coordinate $i \notin S$ is of course the hub containing the hub vertex of the nonhub in the next higher coordinate in $S$.

Finally, to exhibit a weak product of eigensharp graphs failing to be eigensharp, we need an eigensharp graph not in $\mathbf{J}$. There is one on eight vertices.

EXAMPLE 1. Noneigensharp product of eigensharp graphs. Let $G$ be the eightvertex graph of Figure 4, and let $H=K_{3}$. Let the high- and low-degree vertices of $G$ be $U$ and $V$. The signatures of $G$ and $H$ are $(3,1,4)$ (see [1, appendix]) and $(1,0,2) . G$ is in fact star-coverable by stars at $U$. This is the only decomposition of $G$ into 4 complete bipartite subgraphs. If the unique 4-cycle is used, what remains is an 8-cycle and requires 4 more subgraphs. Otherwise, the decomposition uses only stars, and each of the four triangles requires centers of stars on at least two vertices. Doing this in 4 subgraphs forces $U$ to be the set of centers. Thus $G \in \mathbf{J}$ requires a hub/rim decomposition in which the vertices of $U$ are 4 hubs, but it is not possible to partition the edges to the four nonhubs into the three rims required. Hence $G \notin \mathbf{J}$, and $G$ is a candidate for a factor in the desired product.

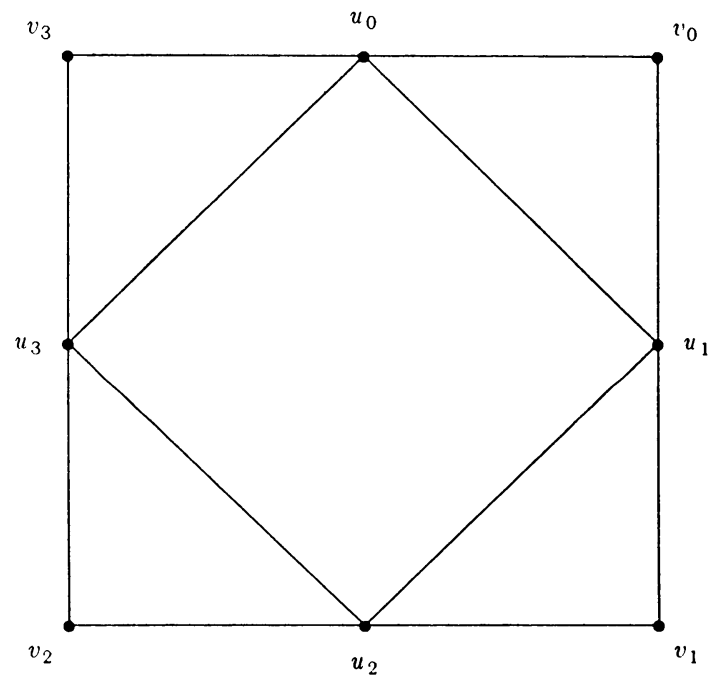

FIGURE 4. The factor $G$ of the noneigensharp weak product in Example 1.

Denote the vertices by $G * H$ by $\left\{u_{j}^{i}\right\} \cup\left\{v_{j}^{i}\right\}$; in each set $0 \leq j \leq 3$ and $0 \leq i \leq 2$. $G * H$ has 72 edges, of which 48 have the form $\left(u_{j}^{i}, v_{k}^{l}\right)$. These edges in fact form a bipartite subgraph $F$ of $G * H$ that is isomorphic to $C_{8} * K_{3}$. One partite set of $F$ has vertices of the form $u_{j}^{i}$, and the other has vertices $v_{k}^{l}$. We claim any complete bipartite subgraph $B$ of $G * H$ has at most 4 edges in $F$. F is symmetric in $u$ and $v$. In one of the partite sets of $B$, only one of the superscripts $0,1,2$ can appear, and on the other side at most two. In either set, the subscripts chosen must all have the same parity, because the corresponding vertices from $G$ must have a common neighbor on the 8-cycle. If only one subscript appears, the partite set has at most 
two vertices. If two different subscripts appear, we can have three or four vertices, but only by using two different superscripts, in which case the other partite set can only have one vertex.

With at most 4 of these edges in each subgraph, $\tau(G * H) \geq 12$. However, $r(G * H)=4 \cdot 2+3 \cdot 1=11$.

\section{REFERENCES}

1. D. M. Cvetkovie, M. Doob, and H. Sachs, Spectra of graphs, Academic Press, New York, 1979.

2. D. M. Cvetković and I. Gutman, The algebraic multiplicity of the number zero in the spectrum of a bipartite graph, Mat. Vesnik 9 (1972), 141-150.

3. R. L. Graham and H. O. Pollak, On embedding graphs in squashed cubes, Graph Theory and Applications (Proc. 2nd Internat. Conf. Graph Theory, Kalamazoo 1972), Lecture Notes in Math., vol. 303, Springer-Verlag, 1973, pp. 99-110.

4. F. Harary, D. F. Hsu, and Z. Miller, The biparticity of a graph, J. Graph Theory 1 (1977), 131-133.

5. A. J. Hoffman, Eigenvalues and partitionings of the edges of a graph, Linear Algebra Appl. 5 (1972), 137-146.

6. L. Lovász, On coverings of graphs, Theory of Graphs (Proc. Conf. Tihany), Academic Press, 1969, pp. 231-236.

7.

8. G. W. Peck, A new proof of a theorem of Graham and Pollak, Discrete Math. 49 (1984), 327-328.

9. B. Reznick, P. Tiwari, and D. B. West, Decomposition of product graphs into complete bipartite subgraphs, Discrete Math. 57 (1985), 179-183.

10. H. Tverberg, On the decomposition of $K_{n}$ into complete bipartite subgraphs, J. Graph Theory 6 (1982), 493-494.

Department of MAThematics, UNiversity OF Illinois, URBana, Illinois 61801 